\title{
Ocular pain and discomfort after advanced surface ablation: an ignored complaint
}

This article was published in the following Dove Press journal:

Clinical Ophthalmology

4 September 2015

Number of times this article has been viewed

\author{
Eva M Sobas' \\ Sebastián Videla ${ }^{2,3}$ \\ Miguel J Maldonado' \\ Jose C Pastor ${ }^{1,4}$ \\ 'Instituto Universitario de \\ Oftalmobiologia Aplicada (IOBA), \\ Universidad de Valladolid, Valladolid, \\ Spain; 'Laboratorios Dr Esteve S.A., \\ Barcelona, Spain; ${ }^{3}$ Department of \\ Experimental and Health Sciences, \\ Faculty of Health and Life Sciences, \\ Universitat Pompeu Fabra, Barcelona, \\ Spain; ${ }^{4}$ Department of Ophthalmology, \\ Hospital Clinico Universitario, \\ Valladolid, Spain
}

Purpose: Laser vision correction is one of the most commonly performed elective surgical procedures in ophthalmology. Generally, discomfort besides pain (photophobia, burning sensation, tearing, and foreign body sensation) after these procedures is not taken into consideration in the clinical practice. The objective is to provide data on these symptoms and their relevance after advanced surface ablation (ASA).

Methods: Single-center survey study based on a structured questionnaire relative to the patients' perceived symptoms after ASA. Inclusion criteria were: $\geq 18$ years old, no ocular disease, with myopia ( 0.75 to $9 \mathrm{D})$ or hyperopia $(0.25$ to $5 \mathrm{D})$ with or without astigmatism, receiving ASA on at least one eye. All procedures were performed by the same surgeon. A descriptive analysis was performed.

Results: Seventy-three consecutive patients (34 men and 39 women) were included in the study. The median (range) of age was 33 (19-64) years. Sixty-nine patients had surgery done on both eyes. Postoperative pain was the most frequent comorbidity (97\% [95\% confidence interval $\{\mathrm{CI}\}$ : 90-100]) with a median (range) of intensity (verbal numerical rating scale) score of 7 (2-10). Photophobia: 85\% (95\% CI: 75-92); burning sensation: 62\% (95\% CI: 50-73); tearing: 59\% (95\% CI: 47-70); and foreign body sensation: 48\% (95\% CI: $36-60)$ were also prevalent postoperative symptoms. Pain during ASA was reported for 44\% (95\% CI: 32-56) of patients.

Conclusion: Comorbidities such as pain, photophobia, burning sensation, tearing, and foreign body sensation are prevalent after ASA procedure. Postoperative pain should be taken into consideration due to its prevalence and intensity. A new and more efficient postoperative analgesic protocol should be established.

Keywords: pain, photophobia, refractive surgery, survey

\section{Introduction}

Advanced surface ablation (ASA) and photorefractive keratectomy (PRK) and its variations are currently common procedures to correct some refractive errors safely and effectively. ${ }^{1}$ Postoperative pain management is one of the drawbacks of these procedures, which remains an unresolved issue..$^{2,3}$

Current studies, focused on finding corneal pain pathways and alternatives to control it, confirm the relevance of this topic. ${ }^{4}$ ASA and the subsequent corneal epithelial defect leave numerous highly sensitive nerve terminals exposed. The stimulation of these terminals results in intense pain and neurogenic inflammation. ${ }^{5}$ With the aim of controlling these effects, researchers have tried some approaches, which are not fully satisfactory. In this vein, although PRK causes less postoperative pain than ASA, severe pain is present in practically all patients after PRK procedure, ${ }^{6}$ which may limit its acceptance. Despite new intrastromal techniques with femtosecond laser which seemed to have reduced this problem, this technique has not been popularized 
yet, probably due to the worldwide recession environment. ${ }^{2,3}$ The mechanism of pain after refractive surgery is multifactorial and its understanding seems crucial for an appropriate management. ${ }^{7}$ Recent papers about pain management in postoperative refractive surgery show that it remains an unsolved issue. , $^{89}$

The potential target for pain relief has numerous approaches; for example, a bandage contact lens (BCL) is effective in improving postoperative comfort by preventing lid movements over the abraded cornea and reducing stimulation of the nerve fibers sensitive to mechanical stimulation. ${ }^{10}$ However, wearing a BCL is not enough to eliminate pain in most patients. ${ }^{11}$ Topical nonsteroidal anti-inflammatory drugs (NSAIDs) help reduce pain following PRK but could interfere with the healing process. ${ }^{12}$ Furthermore, the direct application of cold balanced saline solution (BSS) on the cornea is believed to alleviate the pain caused by exposed nerve endings. ${ }^{12}$

In previously published studies on the evaluation of pain after refractive surgery, some limitations have been observed. One of them is the time of follow-up. In order to make a good assessment of postoperative pain in refractive surgery, a minimum of 72-hour evaluation time is necessary. ${ }^{11,12}$ Studies evaluating pain over a 24 -hour postoperative period are considered insufficient. ${ }^{9}$

Although pain is one of the most important limitations of postoperative refractive surgery, there are other symptoms, generically named discomfort, that have been observed in the majority of patients. These symptoms are another important limitation of refractive surgery, ${ }^{13}$ and have received little attention. Therefore, the purpose of this study is to provide data on the patient-referred ocular sensations in terms of postoperative pain, during and after ASA, evaluated over 1 week, using a numerical rating scale (NRS) and paying attention to other relevant symptoms such as photophobia, burning, tearing, and foreign body sensation. These data will help refractive surgeons to improve their clinical practices.

\section{Patients and methods Study design}

Single-center survey study based on the structured questionnaire relative to the patients' perceived symptoms after ASA. The ethical committee of the University of Valladolid reviewed and approved the study protocol. The study complies with the updated Declaration of Helsinki and the Spanish biomedical research regulatory requirements. All subjects provided their verbal informed consent before their participation in the study.

\section{Study population}

Patients included in this study had to fulfil the following eligibility criteria: $\geq 18$ years old, with no ocular disease other than ametropia, with myopia ( 0.75 to $9 \mathrm{D})$ or hyperopia ( 0.25 to $5 \mathrm{D})$ with or without astigmatism, receiving ASA on at least one eye. All patients were operated on by the same surgeon (MJM). Patients, who fulfilled the inclusion criteria, were identified, and contacted via phone by the same interviewer (EMS). The study was explained to each patient, and before starting the interview, verbal consent was requested.

\section{ASA procedure}

ASA included topical application of one drop of 5\% lidocaine twice at a 10-minute interval, 20 minutes before entering the operating theater. All patients received 0.5 mg alprazolam (Trankimazin ${ }^{\circledR}$, Pfizer, Inc., New York, NY, USA), 30 minutes before surgery. Ethyl alcohol solution (17\%) was placed on the cornea within an 8.5 $\mathrm{mm}$ solution cone and left in place for 30 seconds. Then, a Merocel sponge (Medtronic Xomed Ophthalmics, Inc. Jacksonville, FL, USA) was placed to absorb the excess ethanol. After that, the corneal surface was rinsed with a topical cold BSS. The epithelium was removed with a blunt spatula. This manoeuvre was followed by stromal ablation using excimer laser MEL 70 G (Carl Zeiss Meditec AG, Jena, Germany). This excimer laser incorporates a tracking eye system. The diameter of the treated area was always $6 \mathrm{~mm}$. Then a drop of $0.3 \%$ ofloxacin $\left(\right.$ Exocin $^{\circledR}$, Allergan, Inc., Irvine, CA, USA) and a drop of $0.18 \%$ sodium hyaluronate $\left(\right.$ Vismed $^{\circledR}$, Thea Laboratories, SA, Barcelona, Spain) were applied. A therapeutic contact lens (Acuvue Oasis $^{\circledR}$, Johnson \& Johnson, New Brunswick, NJ, USA) kept at $4{ }^{\circ} \mathrm{C}$ was placed onto the cornea at the end of the procedure. Immediately after surgery, patients received a cold patch for 15 minutes. Patients were given alprazolam $0.5 \mathrm{mg}$ for the first 2 postoperative nights and all other eyedrops (ofloxacin QID, dexamethasone TID, and artificial tears QID) that had to be kept at $4{ }^{\circ} \mathrm{C}$ (fridge temperature). Initially, painkillers were not prescribed unless pain was deemed unbearable. In this case, the patient was given a protocol to use a rescue medication.

\section{Questionnaire: pain intensity and other symptoms after ASA procedure}

An ad hoc questionnaire was created to record the patients' perceived symptoms after ASA (Figure 1). The questionnaire consists of five parts or domains: 1) cognitive orientation, 


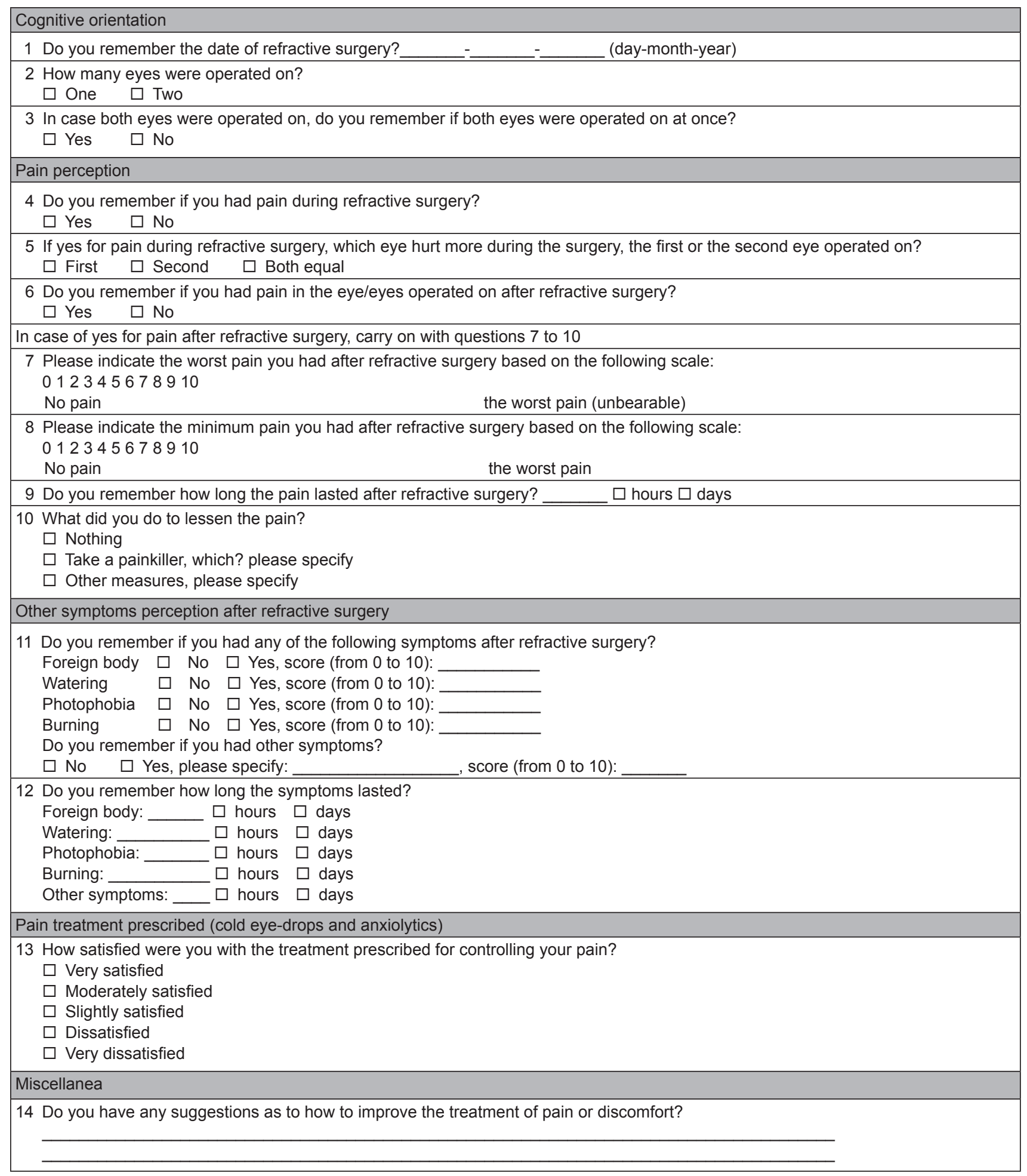

Figure I Ad hoc questionnaire used in the telephonic interview.

2) pain perception, 3) other symptoms (foreign body, tearing, photophobia, burning), 4) pain treatment prescribed, and 5) miscellanea. Furthermore, pain intensity was evaluated by means of an NRS, ie, 0 - no pain and 10- the worst imaginable pain. ${ }^{14}$

\section{Statistical analysis}

This study was designed assuming that 95\% (internal data) of the participants would present pain after ASA procedure. It was calculated that a representative sample for a survey study should include 73 subjects, based on the normal 
approximation with a range of $\pm 2.5 \%$ for a two-sided $95 \%$ confidence interval (CI).

Baseline characteristics were summarized with standard descriptive statistics and a descriptive analysis was carried out. The prevalence of symptoms after ASA procedure was estimated and its 95\% CI was calculated. The Spearman's correlation coefficient was used to assess the relation between the subjective feeling of pain and age and amount of ablation, and between discomfort and age and amount of ablation. Data analysis was carried out using SPSS version 15.0 statistical software (SPSS Inc., Chicago, IL, USA) and StatXact-8 (Cytel Inc., Cambridge, MA, USA).

\section{Results}

\section{Patients' characteristics}

A total of 73 consecutive patients (34 men and 39 women) were included. All contacted patients agreed to participate in the interview. The median (range) of age was 33 (19-64) years, and 69 (95\%) patients received surgery on both eyes.

\section{Pain after ASA procedure}

Pain intensity and other symptoms are shown in Table 1: $51 \%$ of the patients reported pain during surgery; $8 \%$ of the patients reported more pain during the first eye surgery,
$19 \%$ during the second eye surgery, and $73 \%$ of the patients presented the same pain in both eyes. Ninety-seven percent of the patients reported pain (score equal or higher than 2) during the postoperative period. Only two women did not feel pain in the postoperative period. The median (range) score of the worst pain intensity was $7(0-10)$ (mean $[ \pm$ SD]: $6.3[ \pm 2.5])$, and 49 patients $(67 \%)$ had severe pain (6 or higher). It is noteworthy that 38 patients $(52 \%)$ reported a pain intensity higher than 7 . The median (range) duration of the pain was 3 days (range: $1-7)$. Twenty-nine (40\%) patients required analgesic treatment in the postoperative period, out of which eleven patients (15\%; 95\% CI: 8-25) chose analgesic rescue medication and 18 patients $(25 \%)$ used another painkiller (metamizol or ibuprofen), which was not included in the protocol.

Regarding the patients' impression about the prescribed treatment for pain, 67 patients (92\%; 95\% CI: 83-97) scored it as "satisfactory" or "very satisfactory". The remaining six patients marked "slightly satisfactory".

\section{Other symptoms after ASA procedure}

Regarding other postoperative symptoms such as photophobia, burning, tearing, and foreign body sensation, results are shown in Table 1. After pain, the most frequent (85\%) and most intense symptom was photophobia with a mean $( \pm \mathrm{SD})$

Table I Pain intensities (during ASA procedure and postoperative period), photophobia, burning, tearing, and foreign body sensation results

\begin{tabular}{|c|c|c|c|}
\hline Pain and other symptoms & $\begin{array}{l}\text { Overall population } \\
(n=73)\end{array}$ & $\begin{array}{l}\text { Men } \\
(n=34)\end{array}$ & $\begin{array}{l}\text { Women } \\
(n=39)\end{array}$ \\
\hline \multicolumn{4}{|l|}{ Pain during ASA procedure } \\
\hline Yes", number of patients (\%, [95\% Cl]) & $32(44[32-56])$ & $18(53[35-70])$ & $14(36[2 \mid-53])$ \\
\hline \multicolumn{4}{|l|}{ Postoperative pain } \\
\hline Yes", number of patients (\%, [95\% Cl]) & 71 (97 [90-100]) & $34(100[89-100])$ & 37 (95 [83-99]) \\
\hline VRS, median (range)* & $7(2-10)$ & $6(2-10)$ & $7(2-10)$ \\
\hline \multicolumn{4}{|l|}{ Worst postoperative pain } \\
\hline Pain intensity: 2-6, number of patients (\%, [95\% Cl]) & $33(45[34-57])$ & $18(53[35-70])$ & $15(38[23-55])$ \\
\hline Pain intensity: $7-10$, number of patients $(\%,[95 \% \mathrm{Cl}])$ & $38(52[40-64])$ & $16(47[30-65])$ & $22(56[40-72])$ \\
\hline \multicolumn{4}{|l|}{ Photophobia } \\
\hline Yes, number of patients (\%, [95\% Cl]) & $62(85[75-92])$ & 30 (88 [73-97]) & $32(82[66-92])$ \\
\hline VRS, median (range)* & $9(2-10)$ & $8(2-10)$ & $9(2-10)$ \\
\hline \multicolumn{4}{|l|}{ Burning sensation } \\
\hline Yes, number of patients $(\%,[95 \% \mathrm{Cl}])$ & $45(62[50-73])$ & 21 (62 [44-78]) & $24(62[45-77])$ \\
\hline VRS, median (range)* & $7(1-10)$ & $7(2-10)$ & $7(1-10)$ \\
\hline \multicolumn{4}{|l|}{ Tearing } \\
\hline Yes, number of patients (\%, [95\% Cl]) & $43(59[47-70])$ & $20(59[4 I-75])$ & $23(59[42-74])$ \\
\hline VRS, median (range)* & $8(2-10)$ & $8(4-10)$ & $8(2-10)$ \\
\hline \multicolumn{4}{|l|}{ Foreign body sensation } \\
\hline Yes, number of patients $(\%,[95 \% \mathrm{Cl}])$ & $35(48[36-60])$ & $19(56[38-73])$ & $16(4 \mid[26-58])$ \\
\hline VRS, median (range)* & $6(2-10)$ & $5(2-10)$ & $7(2-10)$ \\
\hline
\end{tabular}

Notes: VRS: verbal rating scale (0: no pain; 10: worst imaginable pain). *Pain intensity score of 2 or greater. *The median (range: minimum-maximum) is based only on patients with the symptom.

Abbreviations: ASA, advanced surface ablation; $\mathrm{Cl}$, confidence interval. 
intensity score of $8.0( \pm 2.2)$. Burning sensation was also a very prevalent symptom $(62 \%)$ with a mean $( \pm S D)$ intensity score of $6.8( \pm 2.5)$. It is relevant to mention that 36 patients $(80 \%)$ had values higher than 5 for burning sensation.

The mean $( \pm$ SD) intensity score for tearing was 7.2 $( \pm 2.5)$. A high percentage of patients $(63 \%)$ scored higher than 7 for tearing intensity. The less common symptom was foreign body sensation with a mean $( \pm S D)$ intensity score of $6.1( \pm 2.5) ; 67 \%$ of patients scored equal or higher than 5 for this symptom.

The survey included a question to identify other complaints, referred to by the patients: inability to open eyes (8 [11\%] patients); itching or sharp ocular pain (5 [7\%] patients); swollen eyelids (3 [4\%] patients); dryness (4 [5\%] patients); and migraines (3 [4\%] patients).

No relationship was found between feeling of pain and age and amount of ablation, and between discomfort and age and amount of ablation.

\section{Pain control (cold eye-drop and anxiolytics)}

In this study, chilled BSS was used during surgery and it was recommended that the patient used cold eye-drops to increase analgesia. A protocol of painkillers was not included as a standard in the postoperative medication. ${ }^{15,16}$ Painkillers were used on demand as a rescue medication by the patients themselves. The proportion of patients reporting additional need for analgesia was $40 \%$ (29 patients), but only eleven of them used the recommended rescue medication. The rest of the patients $(25 \%)$ used painkillers not included in the protocol.

\section{Discussion}

ASA and PRK result in a complex cascade of events that trigger sensory afferent fibers in the cornea, producing various degrees of pain. ${ }^{4}$ It is admitted that postoperative pain resulting from corneal epithelial debridement remains a significant limitation to the acceptance and usefulness of these procedures. ${ }^{5,17,18}$ In PRK, epithelial debridement is performed; ${ }^{19}$ thus techniques to preserve the epithelium are used in an attempt to decrease postoperative pain..$^{20}$ But the effect of removal of epithelial flap versus preservation of the flap on postoperative pain is unclear. ${ }^{4}$ In a study, Epi-LASIK versus off-flap Epi-LASIK were compared, and subjective pain scores were lower at 2 hours follow-up in the off-flap Epi-LASIK group, but not at 4, 6, 8, 10 or 24 -hour intervals. ${ }^{21}$ In other study, postoperative pain was similar between EpiLASIK and off-flap Epi-LASIK groups. ${ }^{22}$ Our results are in line with previous studies, in that severe pain was present in practically all patients after ASA procedure, ${ }^{23}$ and the time course of pain was as expected. ${ }^{24}$ Pain begins within 1 hour. Its intensity increases until 4 hours after the surgical procedure, peaking at approximately 24 hours postoperation, ${ }^{25}$ and subsides within 72 hours after surgery, when reepithelialization is complete. ${ }^{24,26}$ Therefore, the results of this study provide evidence that the peak of pain is at 24 hours and median duration of pain is 3 days. In consequence, a complete postoperative pain assessment after ASA should be performed for at least this number of hours.

Management of acute postoperative pain is a challenge for any surgery and acute ophthalmologic postoperative pain is not an exception. Likewise, intraoperative pain is also important. Moreover, data available on the perception of pain during ASA procedure and its frequency are scarce. ${ }^{27}$ Our study evaluated intra-operatory pain finding that half the patients notice pain during surgery. Another novelty in the outcome of this study is that $73 \%$ of these patients referred to the same pain in both eyes and only $19 \%$ reported more pain in the second eye. There are studies reporting the subjectivity of patients' pain after first eye cataract surgery versus second eye, ${ }^{28}$ but we have not found any regarding ASA. Another important result is $25 \%$ of patients used painkillers outside the protocol, acting by themselves instead of using the recommended rescue medication. This lack of compliance was attributed by the patients to the potential side effects related to the prescribed medication.

It is noteworthy that patients who have undergone refractive surgery are generally young and healthy, so they may be considered as "healthy volunteers". Besides, they have been previously informed about the transience of the postoperative discomfort and pain. Therefore, it is fair to say that the benefit of the operation prevails over other negative features. That is to say, the emotional aspect tends to minimize the postoperative symptoms. ${ }^{8}$ Our study did not allow for predicting what type of patients might be at increased risk for pain intolerance. In fact, this could be another area of ocular pain research. To use a questionnaire or other kind of noninvasive test at the time of deciding whether to perform ASA, PRK or another refractive procedure could help the ophthalmologist in the control of postoperative pain. Consequently, this may help minimize the number of patients with severe postoperative pain and discomfort.

A crucial issue dealing with pain is how to measure it in clinical practice. In our study, a verbal NRS was used because the study was conducted via telephone interview. Different types of scales have been developed to evaluate pain, including visual analog scale (VAS) and various categorical scales 
using faces, numbers, or verbal categorical descriptors, ${ }^{29}$ although, none has been specifically developed and validated for ophthalmic pain. ${ }^{17}$ The majority of previous investigators have used general pain scales to measure pain after ophthalmic procedures. ${ }^{23}$ The most recommended methods for the assessment of pain intensity are NRS, verbal rating scale (VRS), or VAS, because all of them provide sufficient power to describe pain intensity. ${ }^{30}$ Most of the reviewed papers used versions of scales according to current recommendations, primarily NRS-11, VRS up to seven categories, and VAS$100 \mathrm{~mm} .{ }^{31} \mathrm{NRS}$ and VRS, which are validated scales, showed a high concordance between them and are equally sensitive in assessing acute pain after surgery. ${ }^{27}$ In a review, these three scales for the assessment of postoperative pain intensity in adults were evaluated. ${ }^{30}$ This study concluded with a novel idea. The most important choice is not the type of scale, but the conditions related to its use, such as a standardized choice of anchor descriptor, methods of administration, and time frame, among others. ${ }^{30}$ They believed that all of these areas can be improved by an international consensus process following the medicine-based evidence rules, which should include improving and standardizing the use of NRS. ${ }^{30}$ The validity of the VAS and NRS for pain could be extended by using other clinically relevant descriptors of pain such as the proportion of patients reporting need for analgesia. ${ }^{32}$ In our study where $97 \%$ of patients reported pain in the postoperative period, only $40 \%$ of patients mentioned that they required analgesics.

Another important limitation of refractive surgery is the existence of postoperative discomfort. ${ }^{26}$ With this respect, a study stood out because the author distinguished between pain and discomfort, explaining that discomfort is a group of symptoms characterized by photophobia, burning, tearing, and foreign body sensation, and all of them were individually evaluated. ${ }^{13}$ Most of the studies only evaluate some of these symptoms, with photophobia being the most frequent..$^{5}$ In a recent study, ${ }^{13}$ most patients reported discomfort with a score of 8 or 9 on a 10-point pain scale, while in our study, photophobia was the only symptom with a score of 8 . After postoperative pain, photophobia was the prevalent symptom present in $85 \%$ of our patients. These results are in line with other reports. . $^{54,25}$

On the other hand, factors such as age and amount of ablation were not associated with pain and discomfort after ASA. Similar findings have been reported. ${ }^{13}$ Although, it is worth mentioning that in our study, all age groups are not represented since the patients included are very young. Therefore, this study suggests that a new pain management strategy is necessary at least during the 3 days postoperative ASA. Similarly, there are other symptoms related to ocular discomfort which should call our attention to lead us toward better postoperative care.

Postoperative pain and discomfort after refractive surgery still is not well controlled. Recent studies have reviewed pain management strategies after PRK. ${ }^{43-35}$ These strategies include the use of cold BSS during the procedure, ${ }^{34}$ and the postoperative placement of extended-wear BCLs until reepithelialization occurs. ${ }^{35}$ Topical NSAIDs are often used to manage pain and discomfort following refractive surgery. ${ }^{4}$ Common ocular adverse events of topical NSAIDs include transient burning, stinging, and conjunctival hyperemia, and the most serious adverse events are superficial punctate keratitis, corneal infiltrates, epithelial defects, and corneal melts. ${ }^{33}$ Moreover, there is also concern that topical NSAIDs may lead to delayed epithelial healing. This was the main reason why these topical drugs were excluded from our ASA postoperative clinical protocol. Instead, oral medications approved for neuropathic pain, such as gabapentin and pregabalin, are adjunctive agents that help minimize the use of topical medication. ${ }^{36}$ Despite the fact that oral NSAIDs are considered excellent analgesics in many acute pain settings, they have not been specifically studied for post-PRK pain. ${ }^{37}$ Oral analgesics, such as opiates, may be used for moderate-to-severe pain after PRK because opioid receptors present in the cornea respond to them. ${ }^{4}$ With the advent of new methods of analgesia targeting specific corneal sensory afferent nociceptors, discomfort can be minimized with less impact on corneal healing. ${ }^{4}$ These concepts might be important to evaluate in future studies looking at optimization of pain management.

There are several limitations to this study, which should be considered before drawing any conclusions. The study design (survey study), the use of a telephone questionnaire instead of a personal interview, and the type of population analyzed (from a single geographical site and operated on by a single surgeon in the center of reference) might underestimate or overestimate the generalization of the results beyond the population and conditions studied. Likewise, since the patients were directly asked about pain, photophobia, burning, tearing, and foreign body sensation, this kind of questioning might overestimate the results. Nevertheless, this type of procedure (telephone questionnaire) in a survey study is considered reliable and valid and has been used for evaluating acute events ${ }^{38,39}$ and chronic pain. ${ }^{40}$ On the other hand, one of the problems related to telephone surveys is their duration, which could influence the quality of the answer. 
This study was done in a 10-minute time period. In spite of these limitations, our study provides new evidences on the comorbidities related to the PRK procedure.

\section{Conclusion}

In conclusion, our results suggest that several comorbidities such as pain, photophobia, burning, tearing, and foreign body sensation are prevalent after an ASA procedure. Among them, postoperative pain should be taken into consideration because of its prevalence and intensity. These results justify additional research to get a more efficient postoperative analgesic protocol after ophthalmologic surgical procedures.

\section{Disclosure}

The authors report no conflicts of interest in this work.

\section{References}

1. Trattler WB, Barnes SD. Current trends in advanced surface ablation. Curr Opin Ophthalmol. 2008;19(4):330-334.

2. Parker J, Tandon A, Shtein RM, et al. Management of pain with diclofenac after femtosecond-assisted laser in situ keratomileusis. J Cataract Refract Surg. 2011;37(3):569-573.

3. Kymionis GD, Kankariya VP, Plaka AD, Reinstein DZ. Femtosecond laser technology in corneal refractive surgery: a review. J Refract Surg. 2012;28(12):912-920.

4. Woreta FA, Gupta A, Hochstetler B, Bower KS. Management of post-photorefractive keratectomy pain. Surv Ophthalmol. 2013;58(6): 529-535.

5. Sher NA, Golben MR, Bond W, Trattler WB, Tauber S, Voirin TG. Topical bromfenac $0.09 \%$ vs ketorolac $0.4 \%$ for the control of pain, photophobia, and discomfort following PRK. J Refract Surg. 2009; 25(2):214-220.

6. Trattler W, McDonald M. Double-masked comparison of ketorolac tromethamine $0.4 \%$ versus nepafenac sodium $0.1 \%$ for postoperative healing rates and pain control in eyes undergoing surface ablation. Cornea. 2007;26(6):665-669.

7. Belmonte C. Eye dryness sensations after refractive surgery: impaired tear secretion or "phantom" cornea? J Refract Surg. 2007;23(6):598-602.

8. Faktorovich EG, Melwani K. Efficacy and safety of pain relief medications after photorefractive keratectomy: review of prospective randomized trials. J Cataract Refract Surg. 2014;40(10):1716-1730.

9. Moshirfar M, Mifflin MD, McCaughey MV, Gess AJ. Prospective, randomized, contralateral eye comparison of tetracaine and proparacaine for pain control in laser in situ keratomileusis and photorefractive keratectomy. Clin Ophthalmol. 2014;8:1213-1219.

10. Cherry PM, Tutton MK, Adhikary H, et al. The treatment of pain following photorefractive keratectomy. J Refract Corneal Surg. 1994; 10(2 Suppl):S222-S225.

11. Cherry PM. The treatment of pain following excimer laser photorefractive keratectomy: additive effect of local anesthetic drops, topical diclofenac, and bandage soft contact. Ophthalmic Surg Lasers. 1996; 27(5 Suppl):S477-S480.

12. Eiferman RA, Hoffman RS, Sher NA. Topical diclofenac reduces pain following photorefractive keratectomy. Arch Ophthalmol. 1993;111(8): 1022.

13. Carones F. Pain management strategies for surface ablation and epiLASIK. Cataract and Refractive Surgery Today. 2007; April:53-54.

14. Breivik H, Borchgrevink PC, Allen SM, et al. Assessment of pain. BrJ Anaesth. 2008;101(1):17-24.

15. Yee RW, Yee SB. Update on laser subepithelial keratectomy (LASEK). Curr Opin Ophthalmol. 2004;15(4):333-341.
16. Stojanovic A, Ringvold A, Nitter T. Ascorbate prophylaxis for corneal haze after photorefractive keratectomy. J Refract Surg. 2003;19(3):338-343.

17. Lee HK, Lee KS, Kim JK, Kim HC, Seo KR, Kim EK. Epithelial healing and clinical outcomes in excimer laser photorefractive surgery following three epithelial removal techniques: mechanical, alcohol, and excimer laser. Am J Ophthalmol. 2005;139(1):56-63.

18. Kanitkar KD, Camp J, Humble H, Shen DJ, Wang MX. Pain after epithelial removal by ethanol-assisted mechanical versus transepithelial excimer laser debridement. J Refract Surg. 2000;16(5):519-522.

19. Griffith M, Jackson WB, Lafontaine MD, Mintsioulis G, Agapitos P, Hodge W. Evaluation of current techniques of corneal epithelial removal in hyperopic photorefractive keratectomy. J Cataract Refract Surg. 1998; 24(8):1070-1078.

20. Camellin M. Laser epithelial keratomileusis for myopia. J Refract Surg. 2003;19(6):666-670.

21. Kalyvianaki MI, Kymionis GD, Kounis GA, Panagopoulou SI, Grentzelos MA, Pallikaris IG. Comparison of Epi-LASIK and off-flap Epi-LASIK for the treatment of low and moderate myopia. Ophthalmology. 2008;115(12):2174-2180.

22. Wang QM, Fu AC, Yu Y, et al. Clinical investigation of off-flap epiLASIK for moderate to high myopia. Invest Ophthalmol Vis Sci. 2008; 49(6):2390-2394.

23. Blake CR, Cervantes-Castaneda RA, Macias-Rodriguez Y, Anzoulatous G, Anderson R, Chayet AS. Comparison of postoperative pain in patients following photorefractive keratectomy versus advanced surface ablation. J Cataract Refract Surg. 2005;31(7):1314-1319.

24. Sher NA, Frantz JM, Talley A, et al. Topical diclofenac in the treatment of ocular pain after excimer photorefractive keratectomy. Refract Corneal Surg. 1993;9(6):425-436.

25. Caldwell M, Reilly C. Effects of topical nepafenac on corneal epithelial healing time and postoperative pain after PRK: a bilateral, prospective, randomized, masked trial. J Refract Surg. 2008;24(4):377-382.

26. McCarty CA, Garrett SK, Aldred GF, Taylor HR. Assessment of subjective pain following photorefractive keratectomy. Melbourne Excimer Laser Group. J Refract Surg. 1996;12(3):365-369.

27. Sipos E, Stifter E, Menapace R. Patient satisfaction and postoperative pain with different postoperative therapy regimens after standardized cataract surgery: a randomized intraindividual comparison. Int Ophthalmol. 2011;31(6):453-460.

28. Adatia FA, Munro M, Jivraj I, Ajani A, Braga-Mele R. Documenting the subjective patient experience of first versus second cataract surgery. $J$ Cataract Refract Surg. 2015;41(1):116-121.

29. Chibnall JT, Tait RC. Pain assessment in cognitively impaired and unimpaired older adults: a comparison of four scales. Pain. 2001;92(1-2): $173-186$.

30. Hjermstad MJ, Fayers PM, Haugen DF, et al. Studies comparing Numerical Rating Scales, Verbal Rating Scales, and Visual Analogue Scales for assessment of pain intensity in adults: a systematic literature review. J Pain Symptom Manage. 2011;41(6):1073-1093.

31. Jensen MP. The validity and reliability of pain measures in adults with cancer. J Pain. 2003;4(1):2-21.

32. Gallagher EJ, Bijur PE, Latimer C, Silver W. Reliability and validity of a visual analog scale for acute abdominal pain in the ED. Am J Emerg Med. 2002;20(4):287-290.

33. Kim SJ, Flach AJ, Jampol LM. Nonsteroidal anti-inflammatory drugs in ophthalmology. Surv Ophthalmol. 2010;55(2):108-133.

34. Niizuma T, Ito S, Hayashi M, Futemma M, Utsumi T, Ohashi K. Cooling the cornea to prevent side effects of photorefractive keratectomy. J Refract Corneal Surg. 1994;10(2 Suppl):S262-S266.

35. Engle AT, Laurent JM, Schallhorn SC, et al. Masked comparison of silicone hydrogel lotrafilcon A and etafilcon A extended-wear bandage contact lenses after photorefractive keratectomy. $J$ Cataract Refract Surg. 2005;31(4):681-686.

36. Pakravan M, Roshani M, Yazdani S, Faramazi A, Yaseri M. Pregabalin and gabapentin for post-photorefractive keratectomy pain: a randomized controlled trial. Eur J Ophthalmol. 2012;22 Suppl 7:S106-S113.

37. Sachs CJ. Oral analgesics for acute nonspecific pain. Am Fam Physician. 2005;71(5):913-918. 
38. Dennis M, Mead G, Doubal F, Graham C. Determining the modified Rankin score after stroke by postal and telephone questionnaires. Stroke. 2012;43(3):851-853.

39. Valle SO, Kuschnir FC, Sole D, Silva MA, Silva RI, Da Cunha AJ. Validity and reproducibility of the asthma core International Study of Asthma and Allergies in Childhood (ISAAC) written questionnaire obtained by telephone survey. J Asthma . 2012;49(4):390-394.
40. Elzahaf RA, Tashani OA, Johnson MI. Prevalence of chronic pain among Libyan adults in Derna city: a pilot study to assess the reliability, linguistic validity, and feasibility of using an arabic version of the structured telephone interviews questionnaire on chronic pain. Pain Pract. 2013; 13(5):380-389.

\section{Publish your work in this journal}

Clinical Ophthalmology is an international, peer-reviewed journal covering all subspecialties within ophthalmology. Key topics include: Optometry; Visual science; Pharmacology and drug therapy in eye diseases; Basic Sciences; Primary and Secondary eye care; Patient Safety and Quality of Care Improvements. This journal is indexed on

\section{Dovepress}

PubMed Central and CAS, and is the official journal of The Society of Clinical Ophthalmology (SCO). The manuscript management system is completely online and includes a very quick and fair peer-review system, which is all easy to use. Visit http://www.dovepress.com/ testimonials.php to read real quotes from published authors. 